\title{
Study of switchgear maintenance somatosensory training system based on ZigBee and LabVIEW techniques
}

\author{
Liu Yaojun, Ding Yamin, Wang Yuyan \\ Shanxi Electric Power Company Training Center \\ Linfen, China \\ 1337967585@qq.com
}

\begin{abstract}
The traditional methods for electrical safety training can not meet the actual needs of the high-voltage, medium-voltage switchgear maintenance and debugging, so this paper proposed a switchgear maintenance somatosensory training system based on ZigBee and LabVIEW technique, which first introduced the somatosensory training in switchgear maintenance training, could enhance safety awareness of trainees, and enhance the effectiveness of training, had an important engineering application value.
\end{abstract}

Keywords—switchgear; somatosensory training; ZigBee; safety training

\section{INTRODUCTION}

With the increasing complexity and intelligence of network structure, requirements of security protection, safety awareness, safety skills for employees of electricity are also increasing. However, the conventional electrical safety training approaches have some deficiencies, and have been unable to fully meet the actual needs of power production safety, which has been particularly evident in the high-voltage, medium voltage switchgear maintenance and debugging process[1].

At present, the maintenance, debugging and training highvoltage, medium-voltage switchgear in our country is mainly carried out by the supply department, the security management have a greater difficulty[2]. However, most of the switchgear maintenance staff gradually upgrade their technology of maintenance and quality with the help of master and his colleagues and theoretical training. But this approach to learning lacks realism, the sense of accident expecting and personal experience feeling, so that maintenance personnel has no intuitive and concrete understanding for the risks during switchgear operation process, and no personal experience about the generated consequences of various erroneous operation[3,4]. Once they start the actual work, the accidents due to improper operation or weak safety awareness will cause great personal injury and property damage.

In response to these problems, this paper have developed a new switchgear maintenance somatosensory training system based on ZigBee and LabVIEW technology, which can achieve 11 somatosensory training projects which can set security risk points randomly, including pulling disconnector with loads, mistakenly going charged interval, installation of charged grounding wire, closing disconnector with ground , etc. in the process of running turning to maintenance or maintenance turning to running. This training system can achieve real-time monitoring of operation process of training course participants, and includes a variety of somatosensory forms like arcing, switch explosions (explosions, smoke, light), switch trip, rushing to open the door, audible alarm, language tips and others to enhance safety awareness of trainee. This training system explores a new ideas about electrical safety education and training to enhance the effectiveness of training, has significant economic benefits and social significance for speeding up the construction of smart grid.

\section{OVERALL ARCHITECTURE OF SWITCHGEAR MAINTENANCE SOMATOSENSORY TRAINING SYSTEM}

Zig Bee technology is a short-range, low-power, low-cost wireless communication technology, mainly used in the field of automatic control, it can easily be embedded in a variety of devices. It is based on IEEE 802.15.4 wireless communication protocol and is easy to implement, it belongs to the low-speed LAN (Personal Area Net-work) standard defined by IEEE. This standard defines the physical layer (PHY) and media access layer (MAC)[5,6]. ZigBee communication protocol has more obvious characteristics and differences compared to other wireless communication protocols like the third generation mobile communication technology (3G), Bluetooth wireless metropolitan area network technology (Wi Max) and so on, such as low cost, easier to achieve, reliable data transmission, short distance, low power consumption and security[7].

Switchgear maintenance somatosensory training system designed the training mode "teaching" $\rightarrow$ "practice" $\rightarrow$ "assessment" $\rightarrow$ "enhanced", the learning process constituted a closed loop. Traditional teacher instilling teaching was upgraded to two-way interactive teaching, which paid more attention to the students' initiative, so that students improved the overall skills through participate behavior rather than raised the level of awareness through classroom participation and experience. 


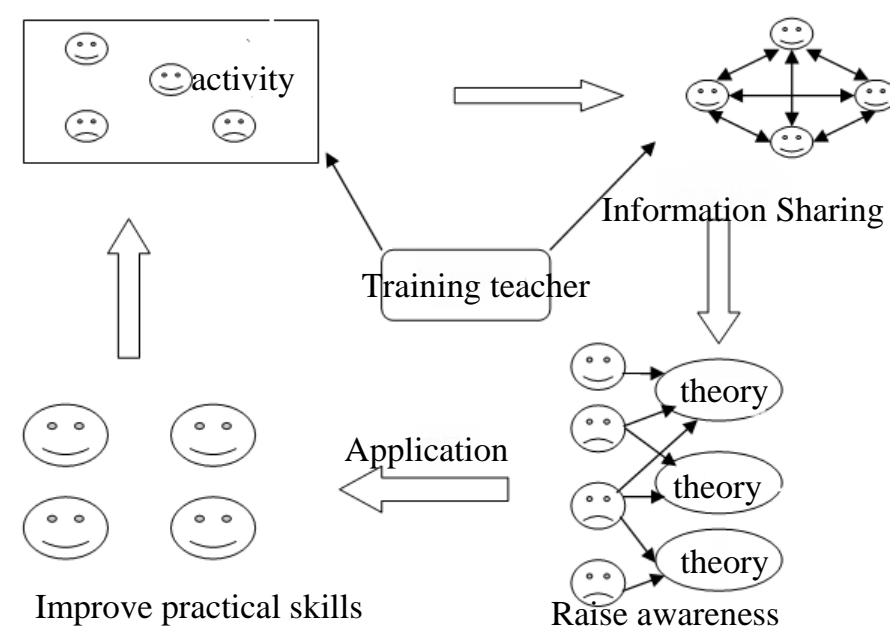

Fig.1 somatosensory training

Switchgear maintenance somatosensory training system consists of data collection, monitoring equipment, somatosensory special effects equipment, system software and other components, shown in Figure2:
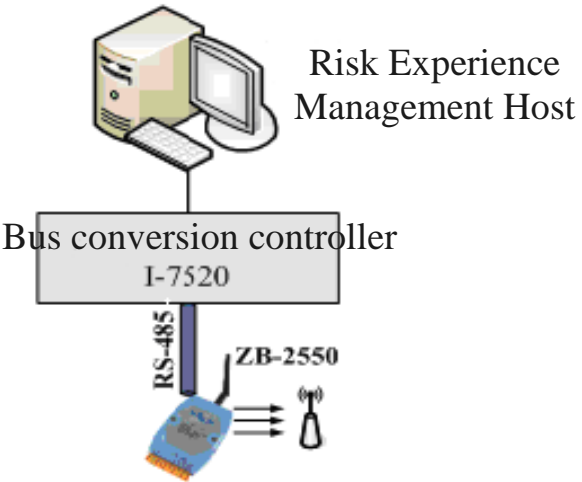

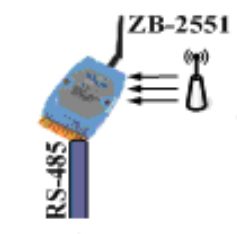

I/O Module

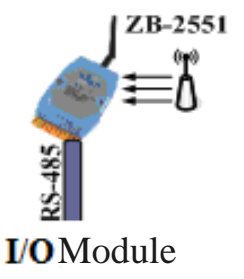

Fig. 2 System Framework

Figure2 shows that the somatosensory training site includes multiple scattered arranged $10 \mathrm{kV}$ switchgears, taking into account the field cabling has some limitations, the entire system uses wireless distributed network structure, information is transfered in the monitoring center and somatosensory training site through the ZigBee wireless transmission module (ZB-2550 and ZB -2551). The system uses a modular design approach to expand the interface and functionality between devices, and manage computers to complete the somatosensory training site management functions, assign experience tasks, recording operation and data query.

During somatosensory training, on-site sensor detects the tool used in the somatosensory operation processes, security supplies status, and device status before and after the operation, represented the operation process by a set of states sequence. Status information was sent to I / O input module (I7051D) by cable transmission. I / O modules connected to controllers by RS485, the controller carried out operated type identification and timing record to determine whether compliance with safety operation specification. According to the type of malfunction, the I / O output module (I-7063D) control simulator of experience effect to generates the accident effect: sound, light, electricity, gas and others.

\section{HARDWARE DEVICES AND SOFTWARE ARCHITECTURE OF SWITCHGEAR MAINTENANCE SOMATOSENSORY TRAINING SYSTEM}

Hardware equipment of switchgear maintenance somatosensory training system includes the training system itself and the input and output device, wherein the somatosensory training system itself is designed on base of the transformation of $10 \mathrm{kV}$ switchgear, input devices mainly are used for real-time monitoring of training participants during the operation, the main physical quantities needed to collected are the position of the switch or a disconnector, whether correct electrical inspection, whether wearing insulated gloves, whether mounting the grounding wire and in the correct location and order, and so on; output equipment is mainly used to achieve special effects, specifically including smoke machines, strobe, light screen sensors, infrared switch sensors and others, the specific effect which can be achieved as follows: door bounce, arc flash, smoke, explosions, shock.

Software development of switchgear maintenance somatosensory training system used graphical test and control platform of LabVIEW virtual instrument to achieve the functions like loop detection and workflow determining of somatosensory training process (as shown in Figure 3). Before trigger event conditions are met, the system software has been in circulation, which periodically reads signal source, refreshes somatosensory signals table and event table. After the trigger event meet the conditions, the system software will judge somatosensory assessment point and choose whether to activate the somatosensory effect set based on the determination result; the training teachers can set risk points in the system dynamically or randomly through software system. 


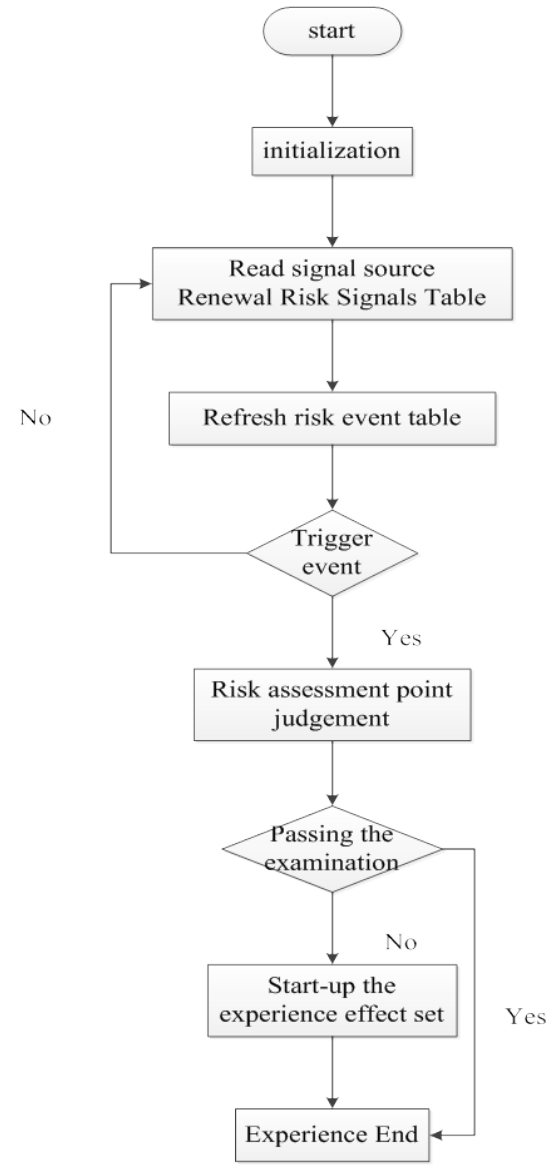

Fig.3 system software flow chart

\section{TYPICAL PROJECTS OF SWITCHGEAR MAINTENANCE SOMATOSENSORY TRAINING SYSTEM}

Based on the actual training needs, system dynamically sets the safety risk points in work of running turn maintenance of switchgear or in contrast, some typical somatosensory training projects are as follows: operated error numbers switchgear; SF6 gas pressure indicated an abnormal position, unchecked; pulled disconnectors with loads; mistakenly charged interval; furnished ground wire charged; unused qualified electrical inspection pen, not wear insulated gloves; not wear insulated gloves, the ground wire installed in the wrong order; the operation of running turning to maintenance is completed without hanging "DO NOT TURN ON, THE LINE IS WORKING" signage; power transmission from the rear end; closed disconnectors with ground; demolished ground without wearing insulated gloves, remove the grounding wire in error sequence.

This paper introduced operational procedures and experience effect taking switchgear running turning to maintenance as a example. The initial state of the switch cabinet is running, so the switch and disconnectors are in the closing position. The first step should be the switchgear power outage operation for the work of running turning to maintenance, the correct procedure is to wear insulated gloves first, then disconnect switches, and finally open the disconnector.
The system first judges whether the operator operates the correct number of switchgear, if operates wrong, error points will be pointed by voice prompt first; then judges whether operating personnel wearing qualified insulated gloves via RFID device, if not, the system voice prompts error points; if the operation sequence has error, which will cause malignant misoperation accident like pulling disconnector with loads, the system triggers the switchgear explosion experience effect including the switch cabinet door bounce, arc flash, smoke, explosions and other effects. If the operator operates power outage of the switchgear right, the next step should be to mount grounding wire. And the correct steps are opening the door, inspecting electricity of the high-voltage terminal first, after confirming no electricity, hanging ground wire according to the order that hanging ground terminal first, and then highvoltage terminal.

When the light curtain sensor detects a person or object enters the cabinet, the system first judges whether highvoltage electrical inspection has been carried out. If electrical inspection has not yet been carried out, it is judged that whether the object is an electrical inspection pen, if not, triggers shock effect, generates arc flash, smoke, explosions and so on.

Operator is required to judge whether the electrical pen is qualified first using use high voltage electroscope before electrical inspection, and then can start the electrical operation. Front-end electrical pen mounts magnet, high voltage terminal of switchgear mounts reed switch, reed switch is closed during the electrical inspection of high voltage terminal to complete electrical inspection operations.

Finally, mount the ground wire. When hanging the ground wire, the system detects whether the attached sequence is correct, if wrong, shock effect is triggered, in addition to the effect of the arc flash, the high-voltage pulse generator of ground wire will be opened for the operator to experience the effects of shock.

\section{CONCLUSION}

In this paper, a new switchgear maintenance somatosensory training system was proposed for the problem that the current switchgear maintenance and debugging lack of effective training methods, which is based on ZigBee wireless communication, LabVIEW virtual instruments and other advanced technologies. The system first introduced the concept of the somatosensory training in switchgear maintenance training fields, and covered eleven typical training programs, had benefits like high degree of integration of hardware and software, intuition, easy to use, and flexiblity. Multiple somatosensory training effects like door bounce, arc flash, smoke, explosions, shock, etc., can further enhance the safety awareness of trainees, enhance the effectiveness of training, have significant economic and social significance for speeding up the construction of smart grid. 


\section{REFERENCES}

[1] Wang shu, Cai li, "perfection of security measures for $10 \mathrm{kV}$ enclosed high voltage switchgear,” Electric Safety Technology, vol. 12, pp. 52-53, 2012.

[2] Zhou kun, “A design for relay protection and automation training simulation system of $220 \mathrm{kV}$ substation main equipment," South China University of Technology, 2013.

[3] Zheng shangci, Zhuang linghui, "Construction and Application of a typical intelligent power distribution switch station Training System,” Electric Power and Electrical Engineering, vol. 4 , pp.68-69+72, Dec. 2009 .
[4] Xie yanbin, Chen zheng, Zhou qian, "Network switchgear security training risks experience system based on the configuration of the wireless,” Electrotechnical Application, vol. S2, pp.504-509, 2013.

[5] Lu zhiyan, "Research of the gas monitoring system for the Coal Mine Based on ZigBee and LabVIEW,” North University, 2014.

[6] Yang wenbo, "Research and development of wireless monitoring system of car model Based on ZigBee and LabVIEW," Tianjin Polytechnic University, 2008.

[7] Zhu mingsheng, Jing hong, "Gesture Tracking and Recognization Based on Motion Sensing Technology,” Computer Systems \& Applications, vol. 8, pp.228-232,2014. 